\title{
Biophysical methods for the study of microbial surfaces
}

\author{
Susana Frases ${ }^{1,2}$ *, Nathan B. Viana ${ }^{3}$ and Arturo Casadevall ${ }^{4}$ \\ ${ }^{1}$ Laboratório de Ultraestrutura Celular Hertha Meyer, Instituto de Biofísica Carlos Chagas Filho, Universidade Federal do Rio de Janeiro, Rio de Janeiro, Brazil \\ ${ }^{2}$ Laboratório de Biologia, Instituto Nacional de Metrologia, Normalização e Qualidade Industrial, Rio de Janeiro, Brazil \\ ${ }^{3}$ Laboratório de Pinças Ópticas-Coordenação de Programas de Estudos Avançados, Instituto de Ciências Biomédicas, Universidade Federal do Rio de Janeiro, \\ Rio de Janeiro, Brazil \\ ${ }^{4}$ Department of Microbiology and Immunology, Albert Einstein College of Medicine, Bronx, NY, USA
}

Edited by:

Marcio Rodrigues, Federal University of Rio de Janeiro, Brazil

Reviewed by:

Tom Coenye, University of Ghent, Belgium

Floyd Layton Wormley, The University of Texas at San Antonio, USA

\section{${ }^{*}$ Correspondence:}

Susana Frases, Laboratório de Ultraestrutura Celular Hertha Meyer, Instituto de Biofísica Carlos Chagas

Filho, Universidade Federal do Rio de Janeiro, llha do fundão, Rio de

Janeiro, Brazil.

e-mail:susanafrases@biof.ufri.br
The challenge in studying the surface architecture of different microbial pathogens is to integrate the most current biochemical, spectroscopic, microscopic, and processing techniques. Individually these methods have insufficient sensitivity to reveal complex structures, such as branched, large, viscous polymers with a high structure hydration, size, and complexity. However, when used in combination biophysical techniques are our primary source of information for understanding polydisperse molecules and complex microbial surfaces. Biophysical methods seek to explain biological function in terms of the molecular structures and properties of specific molecules. The sizes of the molecules found in microbial surfaces vary greatly from small fatty acids and sugars to macromolecules like proteins, polysaccharides, and pigments, such as melanin. These molecules, which comprise the building blocks of living organisms, assemble into cells, tissues, and whole organisms by forming complex individual structures with dimensions from 10 to $10,000 \mathrm{~nm}$ and larger. Biophysics is directed to determining the structure of specific biological molecules and of the larger structures into which they assemble. Some of this effort involves developing new methods, adapting old methods and building new instruments for viewing these structures. The description of biophysical properties in an experimental model where, properties such as flexibility, hydrodynamic characteristics, and size can be precisely determined is of great relevance to study the affinity of the surfaces with biologically active and inert substrates and the interaction with host molecules. Furthermore, this knowledge could establish the abilities of different molecules and their structures to differentially activate cellular responses. Recent studies in the fungal pathogen Cryptococcus neoformans have demonstrated that the physical properties of its unique polysaccharide capsule correlate with the biological functions associated with the intact capsule and the components comprising the capsule. In this review, we describe the application of biophysical techniques to study and characterize this highly hydrated and fragile fungal surface structure.

Keywords: Cryptococcus spp., polysaccharides, optical tweezers, light scattering, zeta potential

\section{INTRODUCTION}

The study of microbial surfaces is critically important because they contain and display components associated with virulence, such as adhesins, and surface structures are often major targets for immune responses. Despite their importance, microbial surfaces are difficult to study because they are often heterogeneous and compositionally complex. Traditional techniques for studying microbial surfaces include electron microscopy and hydrophobicity, both of which can provide important insights into surface structure and composition. In recent years a variety of biophysical techniques have been developed that can be applied to better understand the nature of microbial surfaces. In this essay, we review several techniques that have been applied to study the surface of the pathogenic fungus Cryptococcus neoformans. The importance of this fungus is underscored by the recent report indicating that globally C. neoformans is responsible for approximately one million severe infections and $\sim 600,000$ deaths annually (Park et al., 2011). This organism is unusual because it is surrounded by a polysaccharide capsule and has the distinction of being the only encapsulated human pathogenic fungus (Casadevall and Perfect, 1998). Hence, the distal surface of C. neoformans is essentially that of a gel composed of polysaccharides. Until physical techniques were applied, the polysaccharide surface of $C$. neoformans resisted characterization because it was highly vulnerable to any method used to study. The capsule is composed largely of water and electron microscopy methods that require drying inevitably lead to damage and the likely formation of artifactual fibrillar structures of uncertain relevance to natural capsular structure. In this regard, the more recent biophysical methods applied to this problem studied the capsule and capsular components in hydrated form and avoided concerns resulting from drying. The individual various biophysical techniques each provided important insights that when combined have yielded a new view of capsular architecture. 


\section{BIOPHYSICAL TECHNIQUES AND APPLICATIONS OPTICAL TWEEZERS}

Optical tweezers or optical trap (OT) is a micromanipulation tool that is now widely employed to study mechanical properties in cellular and molecular force scales. It was developed by Arthur Ashkin and his group at the Bell labs (USA) in the early 1970s (Ashkin, 1997). They were initially working on an optical atom trapping experiment and, after encountering certain difficulties in this project, they decided to examine particles in the micron and sub-micron diameter range. The forces involved in the interaction of light with micrometric non-absorbent particles are in the piconewton $\left(10^{-12} \mathrm{~N}\right)$ range. This feature makes OT an appropriate tool to investigate mechanical properties in cellular and molecular scales.

During the first years of development, the attempts to optically trap a particle were performed with counter propagating laser beans, similar to that used in a cooling atom experiment (Ashkin, 1997). In 1987, after placing a Tobacco Mosaic Virus sample in the microscope to test for contamination, they observed that they could isolate and trap contaminant bacteria for many hours by using the laser to illuminate a high numerical aperture lens (Ashkin and Dziedzic, 1987). This result introduced a configuration that by its simplicity became the most used setup for optical tweezers experiments and led to its main application area, biology or mechanical response of biologically related materials.

Although the development of OT started in the 1970s it was only recently that a first principle theory of OT was developed. To date, this theory has been tested by our group and, even though it is not complete, the results indicate confidence in the force measurements performed (Viana et al., 2006, 2007).

\section{Basic optical tweezers applications}

Figure 1 shows the basic schematic representation of a simple optical tweezers setup. A laser emitting light with a wavelength in the infrared spectrum region is used to avoid water absorption of light. The laser is directed to the high numerical aperture microscope objective (MO) lens using a dichroic mirror (DM) of the EPI illumination system of the microscope. After passing by the objective the interaction of the laser light with micrometric dielectric non-absorbent particles in the water sample (WS) produces two forces: the radiation pressure (due to the reflection of the light at the particle in the geometric optics limit) and the gradient force (due to the refraction of the light at the particle in the geometric optics limit). When the gradient force is bigger than the radiation pressure, the particle remains trapped near the focus of the lens. The sample is illuminated by the microscope illumination (MI) system and conveniently positioned by step motors (XY Motor) in the microscope plane of observation. Digitized images of the samples are obtained using a charged coupled device (CCD) camera connected to a computer.

The OT is commonly used to manipulate micrometric objects or as a force sensor in the piconewton range. Recently we developed methodologies using these two features of the OT to measure the viscosity of polysaccharide water solutions in order to study the interaction of yeast cells and to examine the mechanical properties of the capsule.

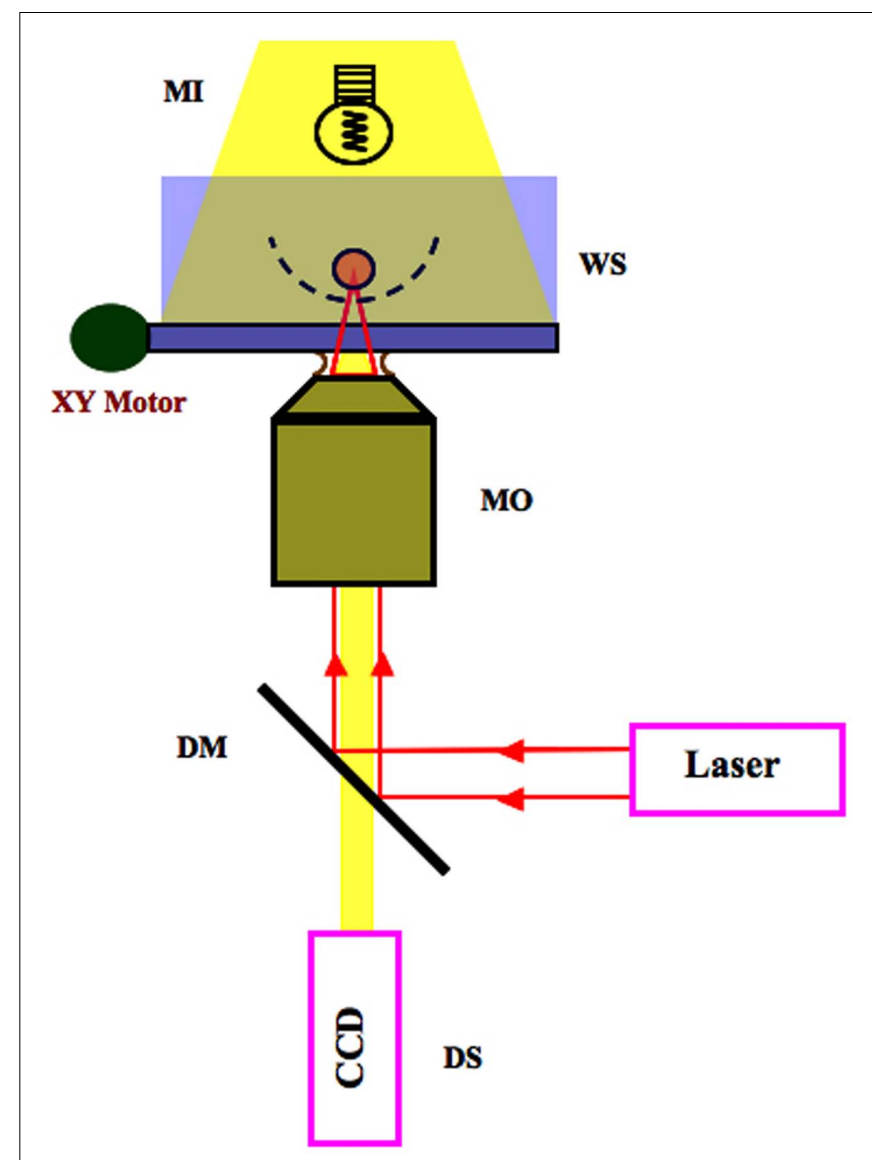

FIGURE 1 | Schematic representation of an optical tweezers setup.

\section{The OT as a micromanipulation tool}

To use the OT as a micromanipulation tool it is usually not necessary to know the force acting on the manipulated object. The microscope positioning system is employed to move the entire sample and to put the objects under the action of the focalized laser light. In this way the object to be trapped can be chosen and positioned as desired by the investigator. Different applications that employ OT in this way are: measurements of viscosity of capsule and algae polysaccharide water solutions, measurement of the Young modulus of the $C$. neoformans capsule and calculation of adhesion time of particles.

\section{LIGHT-SCATTERING TECHNIQUES}

When electromagnetic radiation passes through a given system, the electric field radiation induces a polarization of the molecules that oscillates in time with the same frequency of radiation. The molecules become secondary sources of radiation, and therefore, radiate apart from the incident energy beam. This radiation is called scattered radiation. The frequency changes, the angular distribution, polarization, and intensity of scattered radiation depend on the size, shape, and molecular interactions within the irradiated material. Therefore, from the characteristics of scattered radiation of a given system, it is possible to obtain information on the molecular structure and dynamics of the scattering medium. The most 
common radiation used in scattering experiments is visible light (laser). The choice of the type of radiation is based on the length scale of interest and interaction of radiation with matter.

\section{Static light scattering}

In static light scattering (SLS) the angular dependency of the timemean-intensity of laser light scattered by the particles is measured. The course of the scattered intensity as a function of the detector angle depends on size and structure of the particles. By extrapolating light-scattering measurements made at different angles and concentrations to zero concentration and zero angle we can obtain estimates of the average molecular weight, radius of gyration, and second virial constant without any assumptions.

\section{Dynamic light scattering}

When a laser beam is focused on a large number of particles in solution the light scattered from each particle can be measured by detectors. Since small particles are moving around randomly in the liquid, undergoing diffusive Brownian motion, the distance that the scattered waves travel to a detector varies as a function of time. Electromagnetic waves, like water and sound waves, exhibit interference effects. Scattered waves can interfere constructively or destructively depending on the distances traveled to the detector. The result is an average intensity with superimposed fluctuations. The decay times of the fluctuations are related to the diffusion constants and, therefore, the sizes of the particles. Small particles moving rapidly cause faster decaying fluctuations than large particles moving slowly. The decay times of these fluctuations may be determined either in the frequency domain (using a spectrum analyzer) or in the time domain (using a correlator). The correlator generally offers the most efficient means for this type of measurement. Correlation is defined as the average of the products of the two quantities. From this correlation, effective diameter and polydispersity can be measured. The fluctuating signal, originated from the random motion of particles in a liquid phase and the associated alterations in the intensity of the scattered light over time.

\section{Zeta potential}

Particles dispersed in a liquid often have a charge on the surface. If an electric field is applied in the liquid then these charged particles will move toward either the positive or the negative pole of the applied field. The direction they select is a clear indication of the sign of the charge they carry. The velocity with which they move is proportional to the magnitude of the charge. Zeta potential is calculated from this information. Units of zeta potential are millivolts.

\section{CHARACTERIZATION OF ADHESION TIME BETWEEN MONOCLONAL ANTIBODIES AND HISTOPLASMA CAPSULATUM SURFACE PROTEINS}

This approach was used to characterize the interaction of Histoplasma capsulatum yeast cells in the presence of monoclonal antibodies (mAbs) to an surface expressed heat shock protein of $60 \mathrm{kDa}$ (Hsp60; Guimaraes et al., 2011). The mAbs induced different degrees of yeast cell agglutination that correlated with differences in efficacy in a histoplasmosis model of infection.
CHARACTERIZATION OF C. NEOFORMANS POLYSACCHARIDE The human pathogenic fungus $C$. neoformans has a large polysaccharide (PS) capsule that enlarges during infection and releases copious amounts of PS (exo-PS) into cultures and infected tissues (Casadevall and Perfect, 1998). The capsular PS is a major virulence factor that can elicit protective antibody responses (Casadevall and Perfect, 1998). The capsule is essential for virulence, but the mechanism for capsular growth is unknown. During infection, cryptococcal PS is secreted resulting in deleterious immunological effects such as antibody unresponsiveness (Murphy and Cozad, 1972; Kozel et al., 1977), inhibition of leukocyte migration (Dong and Murphy, 1995), depletion of complement, cytokine production (Vecchiarelli et al., 1995), interference with antigen presentation, and prevention of phagocytosis by macrophages (Kozel and Gotschlich, 1982). Changes in capsular antigenic structure and size have been described and these alterations are associated with the capacity of $C$. neoformans yeast to invade the central nervous system, correlating structural variability with yeast cells crossing the blood-brain barrier. The PS of C. neoformans is the target for experimental PS conjugate vaccines and passive antibody therapies (Pirofski, 2001; Larsen et al., 2005).

The PS that is recovered from culture supernatants has historically provided an ample and convenient source of material for structural and immunological studies. Classical techniques to study polysaccharides, such as serology, did not discriminate structural features of the exo-PS and capsular PS However, a comparison of exo-PS made by two isolation techniques with capsular PS stripped from cells with gamma radiation or dimethyl sulfoxide revealed significant differences in glycosyl composition, mass, size, charge, viscosity, circular-dichroism spectra, and reactivity with mAbs when analyzed by light-scattering techniques (Frases et al., 2008). These tools permitted working with these PS in solution in its native state. The results of these studies strongly suggest that exo-PS and capsular PS are structurally different and this observation has important implications for current views of the relationship between capsular PS and exo-PS for the generation of PS preparations suitable for immunological studies, and for the formulation of PS-based vaccines for the prevention of cryptococcosis (Frases et al., 2008).

In addition, an analysis of capsular PS from cells with small and large capsules by dynamic LS revealed a linear correlation between PS effective diameter and microscopic capsular diameter (Frases et al., 2009). This result implied that capsule growth was achieved by the addition of molecules with larger length, such that some molecules can span the entire diameter of the capsule. Measurement of polystyrene bead penetration of $C$. neoformans capsules by using OT techniques revealed that the outer regions were penetrable, but not the inner regions. These results provided a mechanism for capsular enlargement based on the axial lengthening of PS molecules and suggested the first model for the architecture of a eukaryotic microbial capsule (Frases et al., 2009). In addition, zeta potential experiments demonstrated that aggregation and interaction of PS molecules could be produced by divalent cations which could be one of the capsule formation mediators (Nimrichter et al., 2007).

After determining the basis for the ultra structure of PS capsule, the biophysical techniques allow us to determine the effects 
of carbon sources on the capsule and exo-PS production. For example, growth of $C$. neoformans cells in mannitol significantly increased capsular volume compared with cultivation in glucose (Guimaraes et al., 2010). The fiber lengths and glycosyl composition of capsular polysaccharide from yeast grown in mannitol was structurally different from that of yeast grown in glucose demonstrated by dynamic light scattering (DLS). Combining physical and biological data we demonstrated the capacity of the carbohydrate source and concentration to modify the expression of a major virulence factor of $C$. neoformans. These findings may impact the clinical management of cryptococcosis (Guimaraes et al., 2010).

The capsule of $C$. neoformans is composed of $\beta$-1,4-linked $N$ acetylglucosamine (GlcNAc), and deacetylated glucosamine units play key roles as capsule constituents (Rodrigues et al., 2008). GlcNAc is the monomeric unit of chitin and chitooligomers, which participate in the connection of capsular polysaccharides to the cryptococcal cell wall. Chromatographic and dynamic light-scattering analyses demonstrated that glucuronoxylomannan (GXM), the major cryptococcal capsular component, interacts with chitin and chitooligomers. Interestingly, chitooligomers can form soluble complexes with GXM and interfere with capsular assembly, as manifested by aberrant capsules with defective connections with the cell wall and no reactivity with a mAb to GXM (Fonseca et al., 2009a).

By combining static and DLS, viscosity analysis, and highresolution microscopy we tried to correlate our biophysical findings with biological properties. Analysis of the dependence of capsular PS molecular mass and the radius of gyration provided strong evidence against a simple linear PS configuration. Shape factors calculated from light-scattering measurements in solution revealed values consistent with polymer branching. Furthermore, viscosity measurements by OT provided complementary evidence for structural branching that interfere in complement mediated phagocytosis and inhibit nitric oxide (NO) production (Cordero et al., 2011).

In parallel, analysis of several structural parameters of GXM samples from C. neoformans (serotypes A and D) and Cryptococcus gattii (serotypes B and C) was done in an effort to correlate these variables with the production of $\mathrm{NO}$ by phagocytes and the activation of TLRs (Yauch et al., 2004). TLR-mediated responses were most effectively activated by a PS fraction from a C. gattii serotype B GXM. This serotype B polysaccharide, which was also highly efficient at eliciting the production of NO by macrophages, was similar to the other GXM samples in monosaccharide composition, zeta potential, and electrophoretic mobility. However, immunofluorescence staining using four different mAbs and dynamic light-scattering analysis revealed that the serotype B GXM showed differences in serological reactivity and had the smallest effective diameter among the GXM samples analyzed in

\section{REFERENCES}

Ashkin, A. (1997). Optical trapping and manipulation of neutral particles using lasers. Proc. Natl. Acad. Sci. U.S.A. 94, 4853-4860.

Ashkin, A., and Dziedzic, J. M. (1987). Optical trapping and manipulation

this study. Fractionation of additional serotype B GXMs, followed by exposure of these fractions to macrophages, revealed a correlation between NO production and reduced effective diameters. Our results demonstrate a great functional diversity in GXM samples from different isolates and establish their abilities to differentially activate cellular responses. We propose that serological properties as well as physical chemical parameters, such as the diameter of polysaccharide molecules extracted from DLS, may potentially influence the inflammatory response against Cryptococcus spp. and may contribute to the differences in granulomatous inflammation between cryptococcal species (Fonseca et al., 2010).

The insights learned during the surface characterization of $C$. neoformans capsule using light-scattering techniques, zeta potential, and OT have been applied to other organisms. Description of structural features of Trichosporon asahii surface was done using these methodology. The virulence attributes of T. asahii are virtually unknown, despite its growing relevance as a causative agent of superficial and invasive diseases in humans (Gross and Kan, 2008). Although GXM is a well described virulence factor of pathogenic species in the Cryptococcus genus, it is also produced by species of the Trichosporon genus. Both polysaccharides share antigenic determinants, but unlike cryptococcal GXM, relatively little work has been done on trichosporal GXMs. Analyses of structural and functional aspects of GXM produced by T. asahii and its comparison with the properties of the cryptococcal polysaccharide have revealed that GXM from both species share antigenic reactivity, but PS from T. asahii has a smaller effective diameter and is less negatively charged. These results established that despite similarities in cell wall anchoring, antigenic and antiphagocytic properties, trichosporal and cryptococcal GXMs manifest major structural differences that may directly affect polysaccharide assembly at the fungal surface (Fonseca et al., 2009b).

\section{CONCLUSION}

In summary, biophysical techniques provide powerful means to study certain aspects of microbial surfaces. The application of OT and light scattering has provided new insights into the structure of the C. neoformans capsule and capsular polysaccharide. However, unlike techniques such as crystallography, NMR, and biochemistry that can provide definitive structural information, biophysical approaches often provide a measurement that must be integrated with other information to generate testable models. Hence, biophysical studies constitute a highly intellectual approach to the problem of microbial surfaces whereby information from many different sources must be used to construct a coherent synthesis.

\section{ACKNOWLEDGMENTS}

We thank Joshua Nosanchuk for discussions and assistance with various aspects of this work.

cryptococcal capsular polysaccharides and consequences on its biological activity. Mol. Microbiol. 79, 1101-1117.

Dong, Z. M., and Murphy, J. W. (1995). Effects of the two varieties of Cryptococcus neoformans cells and culture filtrate antigens on neutrophil locomotion. Infect. Immun. 63, 2632-2644.

Fonseca, F. L., Nimrichter, L., Cordero, R. J., Frases, S., Rodrigues, J., Goldman, D. L., Andruszkiewicz, R., Milewski, S., Travassos, L. R., Casadevall, A., and Rodrigues, M.

L. (2009a). Role for chitin and 
chitooligomers in the capsular architecture of Cryptococcus neoformans. Eukaryot. Cell 8, 1543-1553.

Fonseca, F. L., Frases, S., Casadevall, A., Fischman-Gompertz, O., Nimrichter, L., and Rodrigues, $\mathrm{M}$. L. (2009b). Structural and functional properties of the Trichosporon asahii glucuronoxylomannan. Fungal Genet. Biol. 46, 496-505.

Fonseca, F. L., Nohara, L. L., Cordero, R. J., Frases, S., Casadevall, A., Almeida, I. C., Nimrichter, L., and Rodrigues, M. L. (2010). Immunomodulatory effects of serotype B glucuronoxylomannan from Cryptococcus gattii correlate with polysaccharide diameter. Infect. Immun. 78, 3861-3870.

Frases, S., Nimrichter, L., Viana, N. B., Nakouzi, A., and Casadevall, A. (2008). Cryptococcus neoformans capsular polysaccharide and exopolysaccharide fractions manifest physical, chemical, and antigenic differences. Eukaryot. Cell 7, 319-327.

Frases, S., Pontes, B., Nimrichter, L., Viana, N. B., Rodrigues, M. L., and Casadevall, A. (2009). Capsule of Cryptococcus neoformans grows by enlargement of polysaccharide molecules. Proc. Natl. Acad. Sci. U.S.A. $106,1228-1233$.

Gross, J. W., and Kan, V. L. (2008). Trichosporon asahii infection in an advanced AIDS patient and literature review. AIDS 22, 793-795.

Guimaraes, A. J., Frases, S., Cordero, R. J., Nimrichter, L., Casadevall, A., and Nosanchuk, J. D. (2010).
Cryptococcus neoformans responds to mannitol by increasing capsule size in vitro and in vivo. Cell. Microbiol. 12, 740-753.

Guimaraes, A. J., Frases, S., Pontes, B., de Cerqueira, M. D., Rodrigues, $M$. L., Viana, N. B., Nimrichter, L., and Nosanchuk, J. D. (2011). Agglutination of Histoplasma capsulatum by IgG monoclonal antibodies against Hsp60 impacts macrophage effector functions. Infect. Immun. 79, 918-927.

Kozel, T. R., and Gotschlich, E. C. (1982). The capsule of cryptococcus neoformans passively inhibits phagocytosis of the yeast by macrophages. J. Immunol. 129, 1675-1680.

Kozel, T. R., Gulley, W. F., and Cazin, J. Jr. (1977). Immune response to Cryptococcus neoformans soluble polysaccharide: immunological unresponsiveness. Infect. Immun. 18, 701-707.

Larsen, R. A., Pappas, P. G., Perfect, J., Aberg, J. A., Casadevall, A., Cloud, G. A., James, R., Filler, S., and Dismukes, W. E. (2005). Phase I evaluation of the safety and pharmacokinetics of murine-derived anticryptococcal antibody 18B7 in subjects with treated cryptococcal meningitis. Antimicrob. Agents Chemother. 49, 952-958.

Murphy, J. W., and Cozad, G. C. (1972). Immunological unresponsiveness induced by cryptococcal capsular polysaccharide assayed by the hemolytic plaque technique. Infect. Immun. 5, 896-901.

Nimrichter, L., Frases, S., Cinelli, L. P., Viana, N. B., Nakouzi, A., Travassos,
L. R., Casadevall, A., and Rodrigues, M. L. (2007). Self-aggregation of Cryptococcus neoformans capsular glucuronoxylomannan is dependent on divalent cations. Eukaryot. Cell 6, 1400-1410.

Park, S. Y., Love, T. M., Nelson, J., Thurston, S. W., Perelson, A. S. and Lee, H. Y. (2011). Designing a genome-based HIV incidence assay with high sensitivity and specificity. AIDS. PMID: 21716075. [Epub ahead of print].

Pirofski, L. A. (2001). Polysaccharides, mimotopes and vaccines for fungal and encapsulated pathogens. Trends Microbiol. 9, 445-451.

Rodrigues, M. L., Alvarez, M., Fonseca, F. L., and Casadevall, A. (2008). Binding of the wheat germ lectin to Cryptococcus neoformans suggests an association of chitin-like structures with yeast budding and capsular glucuronoxylomannan. Eukaryot. Cell. 7, 602-609.

Vecchiarelli, A., Retini, C., Pietrella, D., Monari, C., Tascini, C., Beccari, T. and Kozel, T. R. (1995). Downregulation by cryptococcal polysaccharide of tumor necrosis factor alpha and interleukin-1 beta secretion from human monocytes. Infect. Immun. 63, 2919-2923.

Viana, N. B., Rocha, M. S., Mesquita, O. N., Mazolli, A., and Maia Neto, P. A. (2006). Characterization of objective transmittance for optical tweezers. Appl. Opt. 45, 4263-4269.

Viana, N. B., Rocha, M. S., Mesquita, O. N., Mazolli, A., Maia Neto, P.
A., and Nussenzveig, H. M. (2007) Towards absolute calibration of optical tweezers. Phys. Rev. E. Stat. Nonlin. Soft Matter Phys. 75(Pt 1), 021914.

Yauch, L. E., Mansour, M. K., Shoham, S., Rottman, J. B., and Levitz, S. M (2004). Involvement of CD14, tolllike receptors 2 and 4, and MyD88 in the host response to the fungal pathogen Cryptococcus neoformans in vivo. Infect. Immun. 72 5373-5382.

Conflict of Interest Statement: The authors declare that the research was conducted in the absence of any commercial or financial relationships that could be construed as a potential conflict of interest.

Received: 24 August 2011; accepted: 17 September 2011; published online: 10 October 2011.

Citation: Frases S, Viana NB and Casadevall A (2011) Biophysical methods for the study of microbial surfaces. Front. Microbio. 2:207. doi: 10.3389/fmicb.2011.00207

This article was submitted to Frontiers in Fungi and Their Interactions, a specialty of Frontiers in Microbiology.

Copyright (c) 2011 Frases, Viana and Casadevall. This is an open-access article subject to a non-exclusive license between the authors and Frontiers Media $S A$, which permits use, distribution and reproduction in other forums, provided the original authors and source are credited and other Frontiers conditions are complied with. 